Nach der vorläufigen Lichtkurve (Tafel 3, Figur 2) scheint $o$ Herculis dem $\beta$ Lyrae-Typus anzugehören. Die Helligkeitsschwankungen sind indessen nur innerhalb der Phasen $\pm 0^{d} .5$ merkbar; in dem übrigen Teil der Periode schwankt die Helligkeit kaum um eine halbe Stufe. Das Spektrum von o Herculis gehört nach Maury der Gruppe VIIb an.

Treptow-Sternwarte, I 9 I I Oktober. o Herculis ist bekanntlich schon früher von $F$. Schwab [A. N. 94.249, vergl. auch A. J. 13.1 $09, \mathrm{Ch}_{2}\left(65^{\circ}\right)$ ] als veränderlich angegeben. $F$. Schwabs Angaben über die scheinbare Periode des Lichtwechsels $\left(6^{d} \cdot 5\right)$, sowie über die Amplitude $\left(o^{\mathrm{m}} \cdot 3\right.$ bis $\left.\circ^{\mathrm{m}} \cdot 4\right)$ stimmen mit obigem recht befriedigend überein.

\title{
Ephemeride des Planeten 480 Hansa
}

berechnet für $\mathrm{I}^{\mathrm{h}} \mathrm{M}$. Z. Berlin nach den Elementen von M. Giacobini.

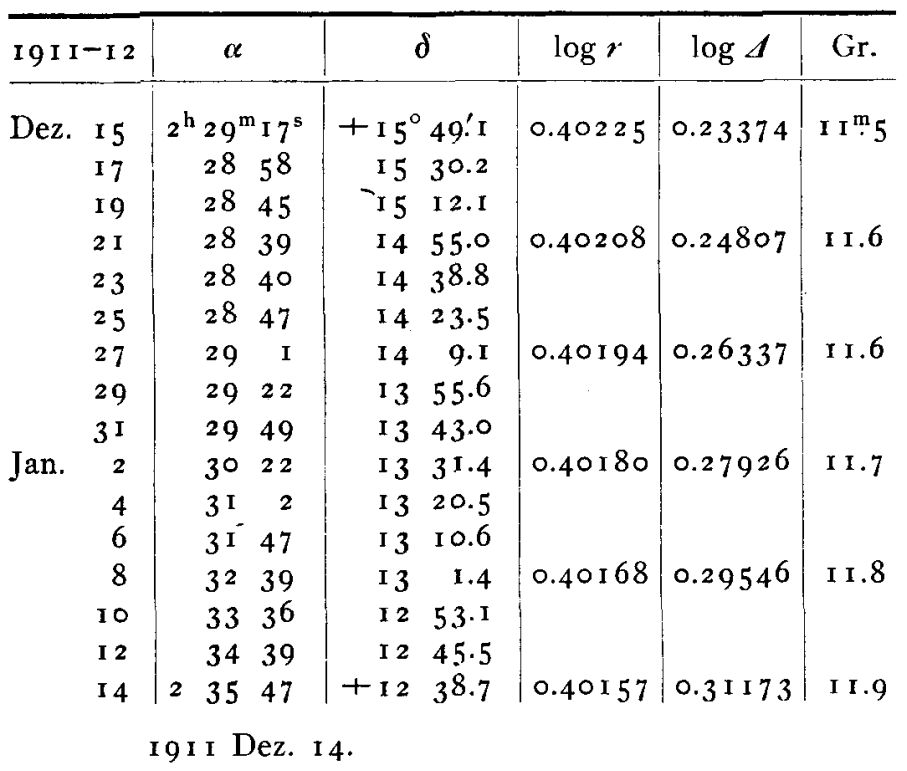

Zuerst unternahm ich die Berechnung der Ephemeride für diesen Planeten nach den Elementen von Bauschinger (Berl. Jahrb. für I9I3) für die Zeit vor und nach der Opposition, welche am 27 . Dezember I 9 I I stattzufinden hätte. $\mathrm{Da}$ die für diesen Tag erhaltenen Werte $\alpha=2.8$ und $\delta=+14^{\circ}$ von den im Berl. Jahrb. angeführten $\alpha=6^{\mathrm{h}} \cdot 4$ und $\delta=+1.6 \mathrm{sehr}$ verschieden sind, könnte man schließen, daß die Opposition bereits Ende Oktober 1. J. stattgefunden hat *). Ich teilte das Ergebnis der nach den Elementen von Bauschinger berechneten Ephemeride Herrn $M$. Giacobini mit, der mir den Rat gab, nochmals die Berechnung der Ephemeride nach den von ihm etwas geänderten Elementen (Astr. Nachr. 4423) vorzunehmen, da seine Elemente eine viel kleinere Differenz zwischen Beobachtung und Rechnung für die Beobachtungen des Planeten im Jahre I 905 und I 906 ergeben. Vielleicht wird man diesen Planeten mittels der vorstehenden Ephemeride auf photographischen Wege noch auffinden können, da er nach I 906 nicht wieder beobachtet wurde.

Dr. Victor Drapczynski, z. Z. Paris, Observatoire.

*) Nach einer Mitteilung von Herrn Professor Berberich müssen die Oppositions-Daten in den Oppositions-Ephemeriden für I9II

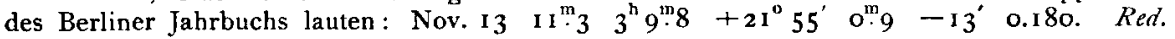

\section{New variable star 86.1911 Lyrae.}

Position: $\quad$ I $855.0 \quad 18^{\mathrm{h}} 19^{\mathrm{m}} 8.7+39^{\circ} 30^{\prime} \cdot 7 \quad$ I $900.0 \quad 18^{\mathrm{h}} 20^{\mathrm{m}} 37^{\mathrm{s}} \cdot 7+39^{\circ} 32^{\prime} \cdot \mathrm{o}$.

The following notes refer to photographic observations of a variable star or nova close to $\mu$ Lyrae. The object is a comparatively faint one - I estimate its maximum

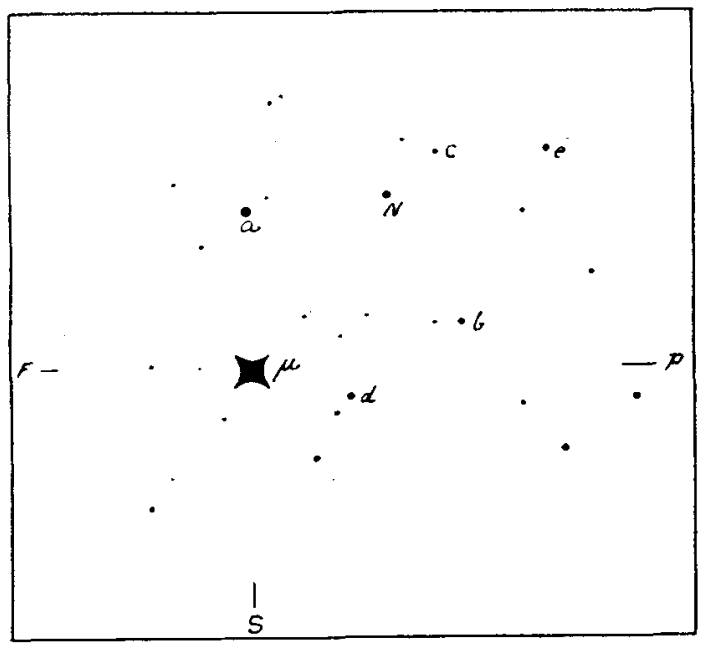

photographic magnitude at $\pm \mathrm{II}^{\text {th }}$ and minimum $<\mathrm{I} 5^{\text {th }}$. For the lettering of the stars in the region about the variable, called $N$, see the accompanying sketch.

The instruments used are $\mathrm{V}=6^{1 / 2}-$ in. Voigtländer Portrait Lens, 30 -in. focus; $D=23 / 4$-in. Dallmeyer Portrait Lens, 8 -in. focus; $R=I^{-i n}$. Reflector, $74.5^{-i n}$. focus.

I9I T Time Plate Remarks

May 25 Io.6 V $4 \quad N$ not visible, $a$ bright, $b$ distinct, $c$ limit of visibility

$\begin{array}{lllll}\text { May } & 27 & 10.3 & V_{5} & \cdot N\end{array}$ position possibly only a defect on the plate, $a$ quite bright, $b$ quite distinct, $c$ visible

June 7 10.0 V $9 \quad N$ not visible, $a$ distinct, $b$ limit of visibility, $c$ not visible

June 8 I0.4 V I I $N$ limit of visibility, $a$ bright, $b$ visible, $c$ visible

Aug. I I 9.2 V I6 $N$ visible, $a$ quite bright, $b$ quite distinct, $c$ distinct

Oct. I $\quad 8.5 \quad \mathrm{D} 29 \quad N$ quite distinct, $a$ quite distinct, $b$ just visible, $c$ extreme limit of visibility 
19I I Time Plate

Oct. I $8^{\mathrm{h}} \cdot 5 \quad \mathrm{~V}_{5} \mathrm{I}$

$\begin{array}{llll}\text { Oct. } & 3^{\mathrm{I}} & 8.8 & \mathrm{~V} \\ 98\end{array}$

Nov. $6 \quad 8.2 \quad R_{30}$

Nov. $96.7 \quad R_{3}$ I $\quad N$ decidedly less bright than $a$ and

Nov. 9 7.9 V I07 $\begin{aligned} & \text { slightly less bright than } d, a \text { quite } \\ & \text { bright, } b \text { bright, } c \text { quite distinct } \\ & \text { slightly less bright than } d, a \text { bright, } \\ & b \text { distinct, } c \text { visible }\end{aligned}$

Nov. 9 7.9 V I07 $\begin{aligned} & \text { slightly less bright than } d \text {, } a \text { quite } \\ & \text { bright, } b \text { bright, } c \text { quite distinct } \\ & \text { slightly less bright than } d, a \text { bright, } \\ & b \text { distinct, } c \text { visible }\end{aligned}$

Nov. 9 7.9 V I07 $\begin{aligned} & \text { slightly less bright than } d \text {, } a \text { quite } \\ & \text { bright, } b \text { bright, } c \text { quite distinct } \\ & \text { slightly less bright than } d, a \text { bright, } \\ & b \text { distinct, } c \text { visible }\end{aligned}$

Nov. 9 7.9 V I07 $\begin{aligned} & \text { slightly less bright than } d \text {, } a \text { quite } \\ & \text { bright, } b \text { bright, } c \text { quite distinct } \\ & \text { slightly less bright than } d, a \text { bright, } \\ & b \text { distinct, } c \text { visible }\end{aligned}$

Nov. 9 7.9 V I07 $\begin{aligned} & \text { slightly less bright than } d \text {, } a \text { quite } \\ & \text { bright, } b \text { bright, } c \text { quite distinct } \\ & \text { slightly less bright than } d, a \text { bright, } \\ & b \text { distinct, } c \text { visible }\end{aligned}$

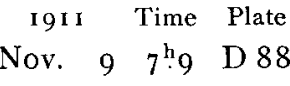
$N$ brighter than $a, a$ quite bright, $b$ bright, $c$ distinct

$N$ slightly less bright than $a, a$ quite bright, $b$ quite distinct, $c$ distinct $N$ decidedly less bright than $a, a$ quite bright, $b$ quite distinct, $c$ distinct. Visual observation at $8^{\mathrm{h}} 45^{\mathrm{m}}$, seeing conditions exceptionally bad, $N$ decidedly brighter than $a$, redish orange in colour
Nov. I $4 \quad 7.0 \quad R_{32}$

Nov. 2 I $\quad 6.5 \quad$ V I I 8

\section{$6.5 \quad \mathrm{D} 94$}

7.2
Remarks

$N$ limit of visibility, $a$ just clearly visible, $b$ and $c$ not visible

$N$ certainly less bright than $d$, but still brighter than $e, a$ bright, $b$ distinct, $c$ visible

$N$ decidedly less bright than $d$ and not brighter than $b$, $a$ quite bright, $b$ quite distinct, $c$ distinct

$N$ extreme limit of visibility, $a$ quite distinct, $b$ visible, $c$ not visible

Visually with the 5 -in. Guiding Refractor $N$ is certainly less bright than $a$, power 70. Air very hazy.

Tatsfield, Surrey, i 9 I I Nov. 24.

\section{Observations de la Comète Borrelly $1905 \mathrm{II}=1911 \mathrm{e}$}

faites à l'Equatorial coudé $(0.32 \mathrm{~m})$ de l'Observatoire d'Alger par MM. C. Rambaud et $N$. Villatte.

\begin{tabular}{|c|c|c|c|c|c|c|c|c|c|c|c|c|}
\hline \multirow{2}{*}{$\frac{19 \text { I I }}{\text { Sept. } 22}$} & \multirow{2}{*}{ 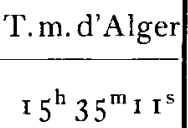 } & \multirow{2}{*}{$\frac{\Delta \alpha}{0^{\mathrm{m}} 43^{\mathrm{s}} \cdot 94}$} & & \multirow{2}{*}{ Cp. } & \multirow{2}{*}{$\begin{array}{l}\text { Obs. } \\
\text { R }\end{array}$} & $\alpha$ app. & \multirow{2}{*}{$\frac{\log p \cdot A}{8.407 n}$} & $\delta$ app. & \multirow{2}{*}{$\mid$\begin{tabular}{c|}
$\log p \cdot d$ \\
$0.9 \pm 6$
\end{tabular}} & \multicolumn{2}{|c|}{ Red. ad 1. app. } & \\
\hline & & & $+3^{\prime} 3$ I.". $^{\prime \prime}$ & & & $3^{\mathrm{h}} 49^{\mathrm{m}} \quad 4^{\mathrm{s}} \cdot 70$ & & $-33^{\circ}$ I I' I 5.'7 & & +2.24 & +28.4 & \\
\hline 23 & I 458 I 3 & 6.17 & $\begin{array}{lll}-- & \text { I } & 47.7\end{array}$ & I 2, I 2 & $\mathrm{R}$ & $\begin{array}{lll}3 & 49 & 54.68\end{array}$ & $9.035 n$ & -33 I $6 \quad 33.6$ & 0.913 & +2.27 & +28.4 & \\
\hline 25 & I 5592 & - 041.74 & +040.8 & I $2, I_{2}$ & $\mathrm{R}$ & $\begin{array}{lll}3 & 51 & 27.90\end{array}$ & $8.75 \mathrm{I}$ & $\begin{array}{lll}-33 & 27 & 16.4\end{array}$ & .916 & $+2.3 I$ & +28.2 & \\
\hline 26 & I $5 \quad 47 \quad 54$ & - I 4.56 & $\begin{array}{lll}-4 & 10.3\end{array}$ & I 2,12 & $\mathrm{R}$ & $3 \quad 52$ 10.1 3 & 8.567 & $\begin{array}{lll}-33 & 3^{2} & 5.2\end{array}$ & 917 & +2.33 & +28.2 & \\
\hline 28 & I 5 I 3 I 6 & +o 10.77 & -1323.0 & I 2, I 2 & $\mathrm{R}$ & $\begin{array}{lll}3 & 53 & 25.51\end{array}$ & $8.5 \mathrm{ron}$ & $-334 \mathrm{I}$ I 8. I & 7 & +2.38 & 8.0 & \\
\hline ct. & I $5 \quad 9 \quad 53$ & to $13 \cdot 36$ & - I 354.3 & I 2, I 2 & $\mathrm{R}$ & $\begin{array}{lll}3 & 55 & 30.04\end{array}$ & 7.8 & $-3357 \quad 6.2$ & 9 I 8 & $+2.5^{\mathrm{I}}$ & +27.4 & \\
\hline 3 & I $5 \quad 22 \quad 36$ & to 24.34 & -1358.7 & I $2, I_{2}$ & $\mathrm{R}$ & $355 \quad 54.28$ & 8.517 & $\begin{array}{lll}-34 & \circ & 2 \\
-3.2\end{array}$ & I 8 & +2 & 6.6 & \\
\hline r 9 & 1 6322 & to 24.88 & -139.6 & 8,6 & V & $355 \quad 41.92$ & 9.442 & $-335^{6} 24 . I$ & & +2 & 4.9 & \\
\hline 20 & $1522 \quad 20$ & 1.77 & 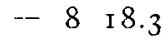 & I 2, I 2 & $\mathrm{~V}$ & $3 \quad 55 \quad$ I 8.86 & 9.292 & -335132.9 & & +2.92 & +24.7 & \\
\hline 23 & I $5 \quad 46 \quad 40$ & to 16.13 & -349.1 & I 2, I 2 & V & $\begin{array}{lll}3 & 53 & 31.47\end{array}$ & 9 & -333148.1 & 0.889 & +2.98 & +24.0 & \\
\hline 26 & $1546 \quad 46$ & $+\mathrm{I} \quad \mathrm{I} 7.67$ & +109.1 & I 2, I 2 & V & 35 I 19.3 I & 9.490 & -33441.9 & 0.882 & +3.06 & +23.4 & \\
\hline 27 & I 54 I 50 & to $27.4 \mathrm{I}$ & $+2 I$ & I 2, I 2 & $\mathrm{~V}$ & $\begin{array}{lll}3 & 50 & 29.07\end{array}$ & 9.487 & $\begin{array}{lll}-32 & 53 & 49 . I\end{array}$ & $0.88 \mathrm{I}$ & +3.08 & $+23 \cdot \mathrm{I}$ & \\
\hline Nov. 3 & I $5 \quad 46 \quad 32$ & 2.94 & $-\circ 17.4$ & I 2, I 2 & V & $343 \quad$ I 1.74 & 9.575 & $74 \mathrm{I} \cdot 5$ & 0.854 & $+3.2 \mathrm{I}$ & $+\mathrm{I} 9.4$ & \\
\hline
\end{tabular}

Positions moyennes des étoiles de comparaison.

\begin{tabular}{|c|c|c|c|}
\hline * & $\alpha 1911.0$ & $\delta$ I9II.0 & Autorité \\
\hline I & $3^{h} 49^{m} 4^{6} \cdot 40$ & $-33^{\circ} 15^{\prime} \mathrm{I} 5^{\prime \prime} \mathrm{I}$ & GZ I 472 \\
\hline 2 & $\begin{array}{lll}3 & 49 & 58.58\end{array}$ & $-33 \quad 15 \quad 14 \cdot 3$ & ) I 48 I \\
\hline 3 & $35^{2}$ & $-3328 \quad 25.4$ & $\gg$ I 553 \\
\hline 4 & $\begin{array}{lll}3 & 53 & 12.36\end{array}$ & $-3328=3.1$ & $\triangleright \quad I 588$ \\
\hline
\end{tabular}

\begin{tabular}{|c|c|c|c|}
\hline$*$ & $\alpha 1911.0$ & $\delta 1911.0$ & Autorité \\
\hline 5 & $3^{\mathrm{h}} 55^{\mathrm{m}}$ I $4^{\mathrm{s}}$ I 7 & $-33^{\circ} 43^{\prime} 39^{\prime \prime} 3$ & Gou 44.32 \\
\hline 6 & $355 \quad 27 \cdot 3^{2}$ & $-3346 \quad 5$ I.I & $\gg 44.37$ \\
\hline 7 & $343 \quad 11.47$ & $\begin{array}{lll}-31 & 7 & 43 \cdot 5\end{array}$ & $\gg 4$ I95 \\
\hline
\end{tabular}

Remarques. Sept. 22. Noyau bien apparent gr. $\mathrm{I}^{\mathrm{m}}{ }^{\mathrm{m}}$. - Sept. 25 . Ciel voilé; les derniers pointés en $\mathscr{A} \delta$ ont été difficiles. - Oct. 2. Nébulosité allongée, $45^{\prime \prime}$ environ; noyau excentré très net de gr. I Im.5. - Oct. I 9 . Nuages; nébulosité avec noyau assez brillant de gr. $\mathrm{I} \mathrm{I}^{\mathrm{m}}$. - Oct. 20 . Observations en $\Lambda \alpha$ difficiles, les deux astres passant en même temps. - Oct. 27. Nébulosité arrondie d'environ $45^{\prime \prime}$ de diamètre; noyau brillant de gr. $10^{\mathrm{m}} .5$.

Observatoire Bouzaréah, I9 I I Déc. 8.

F. Gonnessiat.

\footnotetext{
Inhalt zu Nr. 4544. A. Bemporad. Osservazioni fotometriche della cometa 191 c (Brooks). I 29. - E. D. Roe, Jr. New Double Stars. I37. $\rightarrow$ M. Brendel. Der Planet 699 [19I0 KD]. r37. - H. E. Lau. Notiz betreffend die Veränderlichkeit von 0 Herculis. I39. - $V$. Drapczynski. Ephemeride des Planeten 480 Hansa. I4I. - C. R. D'Esterre. New variable star 86. Iq I Lyrae. 14I. - F. Gonnessiat. Observations de la Comète Borrelly I905 II = I9I I c. I43.
} 